\title{
$X$-INNER AUTOMORPHISMS OF FILTERED ALGEBRAS. II
}

\author{
SUSAN MONTGOMERY ${ }^{1}$
}

\begin{abstract}
We continue earlier work and compute the $X$-inner automorphisms of the ring of differential polynomials in one variable over an arbitrary domain. This is then applied to iterated Ore extensions. We also show that the ring of generic matrices has no nonidentity automorphisms which fix the center
\end{abstract}

In this paper we continue the study of $X$-inner automorphisms of filtered algebras begun in [7], which will be referred to as (I) in what follows. In (I) we described the $X$-inner automorphisms of the enveloping algebra of a Lie algebra, and of the ring of differential polynomials $A=R[x ; d]$ in one variable over a commutative domain $R$. Here we first show that the ring of generic matrices has only the trivial $X$-inner automorphism; equivalently, any automorphism fixing the center is the identity automorphism. We then extend our result from (I) on differential polynomials, allowing an arbitrary prime ring $R$ as the coefficient ring. When $R$ is a domain, we explicitly determine the group of $X$-inner automorphisms of $A=R[x ; d]$ in terms of $R$ and $d$. We then apply these results to certain iterated Ore extensions; in particular, if the original coefficient ring is commutative, then the group of all $X$-inner automorphisms is abelian.

In what follows, $A$ will always denote a prime ring. $\operatorname{Recall}$ that $\sigma \in \operatorname{Aut}(A)$ is $X$-inner if it becomes inner when extended to the (right) Martindale quotient ring $Q_{0}(A)$ of $A$; when $A$ is an Ore domain, $\sigma$ is $X$-inner if and only if it becomes inner on the quotient division ring $D$ of $A$. We shall need the following properties of $Q_{0}(A)$ : it is the (right) quotient ring of $A$ with respect to the filter $\bar{y}$ of all nonzero two-sided ideals (that is, $Q_{0}(A)=\lim _{I \in \text { G. }} \operatorname{Hom}_{A}\left(I_{A}, A\right)$ ), it is a prime ring with center $C=C(A)$ a field, called the extended center of $A$, and $A$ may be imbedded in $Q_{0}(A)$ as left multiplications. By construction, for any $0 \neq x \in A$, there exists a nonzero $I$ of $A$ so that $0 \neq x I \subseteq A$.

Fundamental in what follows is an internal characterization of $X$-inner automorphisms [8].

Lemma 1. If $\sigma \in \operatorname{Aut}(A)$ is $X$-inner, say $r^{\sigma}=s^{-1} r s$, all $r \in A$ for some $s \in Q_{0}(A)$, then there exist nonzero $a, b \in A$ such that $s a=b$ and $a r b=b^{\sigma} r^{\sigma} a$, all $r \in A$. Conversely if arb $=b^{\circ} r^{\circ} a$, all $r \in A$, for some nonzero $a, b \in A$, then there exists $s \in Q_{0}(A)$ with $s a=b$ which induces $\sigma$.

Received by the editors April 23, 1982 and, in revised form. July 19, 1982.

1980 Muthematics Subject Classification. Primary 16A72, 16A38.

${ }^{\prime}$ Research supported in part by NSF grant No. MCS 81-01730. 
Now say that $A$ is filtered; that is, $A=\cup_{n \geqslant 0} A_{n}$, where the $A_{n}$ are additive subgroups of $A, 1 \in A_{0}$, and $A_{n} A_{m} \subseteq A_{n+m}$, all $n, m$. For any $a \in A$, let $f(a)$ denote the filtration of $a$ (that is, $f(a)=n$ if $a \in A_{n}$ but $a \notin A_{n-1}$ ). We say that $f$ is a degree function if $f(a b)=f(a)+f(b)$, all $a, b \in A$. The next lemma is essentially Proposition 1, part (1), of (I).

LEMMA 2. Let $A$ be filtered with a degree function $f$. If $\sigma$ is an X-inner automorphism of $A$, then $f\left(a^{\circ}\right)=f(a)$, all $a \in A$.

We first consider the ring of generic matrices, and show that it has a degree function. For general facts about PI rings, we refer the reader to [3]. Let $U=$ $k\left\{X_{1}, \ldots, X_{m}, \ldots\right\}$ denote the ring of $n \times n$ generic matrices over an infinite field $k$. We may identify $U$ with $F / T_{n}$, where $F=k\left\{y_{1}, \ldots, y_{m}, \ldots\right\}$ is the free algebra and $T_{n}$ is the $T$-ideal of $F$ of all identities of $n \times n$ matrices. $F$ is a graded algebra using the usual degree function $d$; that is, $F=\Sigma_{m \geqslant 0} \oplus F_{m}$, where $F_{m}$ is the $k$-space spanned by the homogeneous monomials of degree $m$. If $k$ is an infinte field, a standard argument shows that $T_{n}$ is a graded ideal (if $p=\Sigma p_{m} \in T_{n}$, where $p_{m} \in F_{m}$, replace each $y_{i}$ by $\lambda y_{i}, 0 \neq \lambda \in k$, and use a Vandermonde determinant to see that $p_{m} \in T_{n}$ ). Thus $U=F / T_{n}$ is also a graded algebra, where for any $0 \neq a \in U$, the filtration of $a$ is given by $f(a)=\min \left\{d(p) \mid a=p+T_{n}, p \in F\right\}$.

Now $U$ is a domain by the theorem of Amitsur (see [3, p. 90]). In a graded ring which is a domain, the filtration is additive, and so is a degree function. We summarize the above remarks as a lemma.

LeMma 3. Let $U=F / T_{n}$ be the ring of generic matrices over an infinite field $k$. Then $U$ has an (additive) degree function which is induced from the degree function in $F$.

THEOREM 1. Let $U=k(X)$ be the ring of generic matrices over an infinite field $k$. If $\sigma \in \operatorname{Aut}(U)$ fixes the center of $U$, then $\sigma=1$.

Proof. By Posner's theorem, $U$ has a quotient division ring $D$; so $D=U Z^{-1}$, where $Z$ is the center of $U$, and $C$, the center of $D$, is just the quotient field of $Z$ [3]. Thus $\sigma$, when extended to $D$, fixes $C$, so is inner on $D$ by the Skolem-Noether theorem. Since $D=U Z^{-1}$, we may assume that $\sigma$ is induced by an element $a \in U$.

Now for each generic matrix $X_{i}, a X_{i}^{\sigma}=X_{i} a$. Using the degree function in the lemma and the fact that $f\left(X_{i}\right)=1$, we see that $f\left(X_{i}^{\boldsymbol{\sigma}}\right)=1$ also. Thus $X_{i}^{\boldsymbol{\sigma}}=$ $\sum_{j=1}^{m} a_{j}+\gamma I$, some $\alpha_{j}, \gamma \in k$.

Since $a^{-1} \in D, a$ is regular in $U$, and so $a$ is also invertible considered as a matrix in $M_{n}\left(k\left(x_{i j}^{l}\right)\right)$, where the $\left\{x_{i j}^{l}\right\}_{l=1.2 \ldots}$ are the entires of the generic matrices (see [1, Lemma 10.3]). Thus we can view $X_{i}^{\sigma}=a^{-1} X_{i} a$ as an equation in matrices; it follows that $\operatorname{tr}\left(X_{i}^{\sigma}\right)=\operatorname{tr}\left(X_{i}\right)$ and $\operatorname{Det}\left(X_{i}^{\sigma}\right)=\operatorname{Det}\left(X_{i}\right)$.

Then $\operatorname{tr}\left(X_{i}^{o}\right)=\sum_{j=1}^{m} \alpha_{j} \operatorname{tr}\left(X_{j}\right)+n \gamma=\operatorname{tr}\left(X_{i}\right)$ implies that $\alpha_{i}=1$ and $\alpha_{j}=0$ if $j \neq i$, since $\sum_{l=1}^{n} x_{l l}^{i}$ is algebraically independent over $k$ from $\sum_{l=1}^{n} \sum_{j=1, j \neq i}^{m} x_{l l}^{j}$. Moreover $n \gamma=0$. Thus $X_{i}^{\sigma}=X_{i}+\gamma I$. But now, since $\operatorname{Det}\left(X_{i}^{0}\right)=\operatorname{Det}\left(X_{i}+\gamma\right)=$ $\operatorname{Det}\left(X_{i}\right)$, it follows that $\gamma=0$. That is, $X_{i}^{\sigma}=X_{i}$, for all $i$. We have shown that $\sigma=1$.

As a consequence of Theorem 1 , if $G$ is any finite group of automorphisms of the generic matrix ring $U$, then the fixed ring $U^{G}$ has the same pi degree as $U[5$, Theorem 4.2]. 
We now turn to differential polynomial rings. Let $R$ be a prime ring with 1 with a derivation $d$, and let $A=R[x ; d]$, the differential polynomial ring in which $x r=r x$ $+d(r)$, all $r \in R$. In the case when $R$ is a commutative domain, the $X$-inner automorphisms of $A$ were described in (I), Theorem 2. Namely, let $F$ be the quotient field of $R$, and $D$ the quotient division ring of $A$ (as $A$ is an Ore domain in this case), and let $\sigma \in \operatorname{Aut}(A)$. If $\sigma$ becomes inner on $D$, say induced by $s=b(x) a(x)^{-1} \in D$, then $\sigma$ is also induced by $q=\beta_{n} \alpha_{m}^{-1} \in F$, where $\beta_{n}$ and $\alpha_{m}$ are the leading coefficients of $b(x)$ and $a(x)$ respectively. In particular it follows that $s=c q$, where $c$ is in the center of $D$.

We show here that a similar result holds when the coefficient ring $R$ is any domain; in fact something can be said when $R$ is prime. Our argument is an extension of the proof in (I). Thus, consider an $X$-inner automorphism $\sigma$ of $A=R[x ; d]$, for $R$ prime. $A$ is filtered, using $A_{n}=$ all polynomials of degree $\left.\leqslant n\right\}$, and we let $f(p)$ denote the "degree" of any $p \in A$. If $R$ is a domain, then $f(p q)=f(p)+f(q)$, all $p, q \in A$; thus by Lemma $2, \sigma$ preserves the degree of any polynomial $p \in A$. Now by Lemma $1, \sigma$ is induced by some unit $s \in Q_{0}(A)$, and there exist polynomials $a=a(x), b=b(x) \in A$ so that $s a=b \neq 0$ and

$$
a p b=b^{\sigma} p^{\sigma} a, \quad \text { all } p \in A \text {. }
$$

Write $a=\alpha_{m} x^{m}+\cdots+\alpha_{0}$ and $b=\beta_{n} x^{n}+\cdots+\beta_{0}, \alpha_{i}, \beta_{i} \in R$.

TheOREM 2. Let $A=R[x ; d]$, where $R$ is prime with derivation $d$. Let $\sigma$ be an $X$-inner automorphism which preserves the degree of any polynomial. Let $s \in Q_{0}(A)$ induce $\sigma$, and let $a, b \in A$ be as described above. Then $\sigma$ is also induced by $q \in Q_{0}(R)$, where $q \alpha_{m}=\beta_{n} \neq 0$ and $\alpha_{m} p \beta_{n} \neq \beta_{n}^{\sigma} p^{\sigma} \alpha_{m}$, all $p \in A$. Moreover,

(1) $x^{\sigma}=x+\alpha$, where $\alpha=q^{-1} d(q)=s^{-1} d(s) \in R$,

(2) $q$ may be identified with an element of $Q_{0}(A)$, so that $s=c q$, where $c \in C(A)$, the extended center of $A$.

Proof. Since $\sigma$ preserves degree, $x^{\sigma}=\beta x+\alpha, \alpha, \beta \in R$. We claim that $\beta=1$. For, choose any $r, t \in R$ and use $p=r x t$ in (*):

$$
a(x)(r x t) b(x)=b(x)^{\sigma} r^{\sigma}(\beta x+\alpha) t^{\circ} a(x) .
$$

Equating coefficients of highest degree $n+m+1$, we see that $\alpha_{m} r t \beta_{n}=\gamma_{n} r^{\sigma} \beta t^{\sigma} \alpha_{m}$, where $b(x)^{\sigma}=\gamma_{n} x^{n}+\cdots+\gamma_{0}$. Using $p=r t$ in (*), we get $\alpha_{m} r t \beta_{n}=\gamma_{n} r^{\sigma} t^{\sigma} \alpha_{m}$. Thus $\gamma_{n} r^{\sigma}(\beta-1) t^{\sigma} \alpha_{m}=0$, all $r, t \in R$. Since $R$ is prime, it follows that $\beta=1$. Thus $x^{0}=x+\alpha$.

It then follows that the highest coefficient of $b(x)^{\sigma}$ is just $\beta_{n}^{o}$, for $b(x)^{\sigma}=$ $\beta_{n}^{o}(x+\alpha)^{n}+\cdots+\beta_{0}^{o}$. Now using $p=r \in R$ in $(*)$ and equating highest degree terms,

$$
\alpha_{m} r \beta_{n}=\beta_{n}^{\circ} r^{\circ} \alpha_{m}, \quad \text { all } r \in R .
$$

That is, $\sigma$ is $X$-inner on $R$, with the desired $\alpha_{m}, \beta_{n}$. Let $q \in Q_{0}(R)$ be a unit so that $q \alpha_{m}=\beta_{n}$ and so $q$ induces $\sigma$ on $R$. We will show that $q$ induces $\sigma$ on $A$.

We require two equations. For the first, equate coefficients of degree $n+m-1$ of $a r b=b^{\circ} r^{o} a$, to obtain

$$
m \alpha_{m} d\left(r \beta_{n}\right)+\alpha_{m-1} r \beta_{n}+\alpha_{m} r \beta_{n-1}=n \beta_{n}^{o} d\left(r^{o} \alpha_{m}\right)+\gamma_{n-1} r^{\sigma} \alpha_{m}+\beta_{n}^{o} r^{o} \alpha_{m-1}
$$


For the second we compare coefficients of terms of degree $n+m$ in $a(r x t) b=$ $b^{\sigma} r^{\circ}(x+\alpha) t^{\circ} a$. For the left-hand side we have

$$
\begin{aligned}
\left(\alpha_{m} x^{m}+\alpha_{m-1} x^{m-1}+\cdots\right)(r x t)\left(\beta_{n} x^{n}+\beta_{n-1} x^{n-1}+\cdots\right) & \\
=\alpha_{m} r t \beta_{n} x^{m+n-1}+\left[\alpha_{m} r(m+1) d\left(t \beta_{n}\right)\right. & +\alpha_{m} m d(r) t \beta_{n} \\
& \left.+\alpha_{m-1} r t \beta_{n}+\alpha_{m} r t \beta_{n-1}\right] x^{n+m}+\cdots
\end{aligned}
$$

That is, the degree $n+m$ coefficient is

$$
m \alpha_{m} d\left(r t \beta_{n}\right)+\alpha_{m} r d\left(t \beta_{n}\right)+\alpha_{m-1} r t \beta_{n}+\alpha_{m} r t \beta_{n-1} .
$$

For the right-hand side,

$$
\left(\beta_{n}^{o} x^{n}+\gamma_{n-1} x^{n-1}+\cdots\right)\left(r^{o}(x+\alpha) t^{\sigma}\right)\left(\alpha_{m} x^{m}+\alpha_{m-1} x^{m-1}+\cdots\right)
$$

gives as the degree $n+m$ coefficient

$$
n \beta_{n}^{\sigma} d\left(r^{\sigma} t^{\sigma} \alpha_{m}\right)+\beta_{n}^{\sigma} r^{\sigma} d\left(t^{\sigma} \alpha_{m}\right)+\beta_{n}^{\sigma} r^{\sigma} t^{\sigma} \alpha_{m-1}+\gamma_{n-1} r^{\sigma} t^{\sigma} \alpha_{m}+\beta_{n}^{\sigma} r^{\sigma} \alpha t^{\sigma} \alpha_{m} .
$$

Now, using $(3)=(4)$, and cancelling terms using (2) with $r$ replaced by $r t$, we obtain our second equation

$$
\alpha_{m} r d\left(t \beta_{n}\right)=\beta_{n}^{\sigma} r^{\sigma} d\left(t^{\sigma} \alpha_{m}\right)+\beta_{n}^{\sigma} r^{\sigma} \alpha t^{\sigma} \alpha_{m} .
$$

We are now able to show the crucial fact that $d(q)=\alpha q$. For, since $q \alpha_{m}=\beta_{n}$, $q \alpha_{m} q=\beta_{n} q=q \beta_{n}^{\sigma}$, and so $\alpha_{m} q=\beta_{n}^{o}$. Also, for any $r \in R, \alpha_{m} r q=\alpha_{m} q r^{o}=\beta_{n}^{o} r^{o}$. Substituting in (5),

$$
\alpha_{m} r d\left(t \beta_{n}\right)=\alpha_{m} r q d\left(t^{\sigma} \alpha_{m}\right)+\alpha_{m} r q \alpha t^{\sigma} \alpha_{m} .
$$

That is, $\alpha_{m} r\left(d\left(t \beta_{n}\right)-q d\left(t^{\sigma} \alpha_{m}\right)-q \alpha t^{\sigma} \alpha_{m}\right)=0$, all $r, t \in R$. Since $R$ is prime, it follows that $d\left(t \beta_{n}\right)=q d\left(t^{\sigma} \alpha_{m}\right)+q \alpha t^{\circ} \alpha_{m}$. But now $t \beta_{n}=t q \alpha_{m}=q t^{\circ} \alpha_{m}$, and thus $d\left(t \beta_{n}\right)=d(q) t^{\circ} \alpha_{m}+q d\left(t^{\circ} \alpha_{m}\right)$. Comparing with the previous equation, we get $d(q) t^{\sigma} \alpha_{m}=q \alpha t^{\sigma} \alpha_{m}$, all $t \in R$. But then $(d(q)-q \alpha) R \alpha_{m}=0$; since $R$ is prime, $d(q)=q \alpha$.

Now, the derivation $d$ on $R$ extends to a derivation on $Q_{0}(R)$ (see [4]) so we may consider the ring $B=Q_{0}(R)[x ; d]$, on which $\sigma$ is also an $X$-inner automorphism (as it extends to $Q_{0}(R)$ naturally and is induced on $Q_{0}(R)$ by $q$ ). We claim that $q$ induces $\sigma$ on all of $B$. For, we know that $q$ induces $\sigma$ on $Q_{0}(R)$, and $q^{-1} x q=$ $q^{-1}(q x+d(q))=x+q^{-1} d(q)=x+\alpha=x^{\sigma}$, using $d(q)=q \alpha$ from above. Since $B$ is generated by $Q_{0}(R)$ and $x, q$ induces $\sigma$ on all of $B$.

Now, since $q \alpha_{m}=\beta_{n}$, it follows that $\alpha_{m} p \beta_{n}=\beta_{n}^{o} p^{o} \alpha_{m}$, for all $p \in B$. For, $q p^{\circ}=p q$, so $\alpha_{m} q p^{\sigma} \alpha_{m}=\alpha_{m} p q \alpha_{m}$. Replacing $\alpha_{m} q$ by $\beta_{n}^{\circ}$, the desired equation follows.

It remains only to show that we may identify $q$ with an element of $Q_{0}(A)$. Now since $q \in Q_{0}(R)$, there exists a nonzero ideal of $R$ with $0 \neq q I \subseteq R$. Let $I^{\prime}=$ $\sum_{i=0}^{\infty} d^{\prime}(I)$. Then $I^{\prime}$ is also an ideal of $R$. For, say $t \in d^{n}(I)$, and $r \in R$. Now $t=d(u)$, some $u \in d^{n-1}(I)$, and $d(u r)=d(u) r+u d(r)$; thus $t r=d(u r)-u d(r)$. By induction on $n, u r \in I^{\prime}$, which is $d$-stable, so $d(u r) \in I^{\prime}$. Again by induction, $u d(r) \in I^{\prime}$; thus $t r \in I^{\prime}$, so $I^{\prime}$ is a $d$-stable ideal of $R$. We also claim that $q I^{\prime} \subseteq R$. Again proceed by induction, and assume that $q d^{i}(I) \subseteq R$, all $i=0, \ldots, n-1$. Choose $t \in d^{n}(I)$; then $t=d(u)$, some $u \in d^{n-1}(I)$, so $q u \in R$ by induction. Since 
$d(q u)=d(q) u+q d(u)=q \alpha u+q t, q t=d(q u)-q \alpha u=d(q u)-\alpha^{\sigma^{-1}} q u \in R$. Thus $q I^{\prime} \subseteq R$.

Let $J=I^{\prime}[x]$. Since $I^{\prime}$ is $d$-stable, $J$ is an ideal of $A$. Finally, since $q I^{\prime} \subseteq R$, $q J \subseteq A$. Thus $q$ determines a right $A$-module homomorphism $J \rightarrow A$, so may be identified with an element of $Q_{0}(A)$.

Since $q$ and $s$ are both in $Q_{0}(A)$ and induce $\sigma$, there exists some $c \in C(A)$ so that $s=c q$. The theorem is proved.

We remark that if $R$ is a domain, the theorem applies to all $X$-inner automorphisms of $A$, since (as noted before the theorem) in that case any $X$-inner automorphism preserves the degree of a polynomial.

If $R$ is not a domain, however, then $X$-inner automorphisms do not necessarily preserve degree, and cannot be induced by an element of $Q_{0}(R)$. For example. let $R$ be any prime ring which is not a domain, and let $A=R[x]$, the ordinary polynomial ring. $R$ contains an element $\alpha \neq 0$ with $\alpha^{2}=0$; thus $s=1+\alpha x$ is a unit in $A$. Let $\sigma$ be conjugation by $s ; \sigma$ is certainly $X$-inner. But $\sigma$ does not preserve degree: as $R$ is prime we may choose $\beta \in R$ so that $\alpha \beta \alpha \neq 0$. But then $\beta^{\circ}=(1-\alpha x) \beta(1+\alpha x)=$ $\beta+(\beta \alpha-\alpha \beta) x-\alpha \beta \alpha x^{2}$, which has degree 2 .

We now wish to give an explicit description of the group of $X$-inner automorphisms of $R[x ; d]$ when $R$ is a domain; to do this, we require some notation. As defined in [8], let $N(R)=\left\{s \in Q_{0}(R) \mid s R=R s\right\}$, the normalizing elements of $R$ in $Q_{0}(R)$, and let $N^{*}(R)$ denote the nonzero elements of $N(R)$. Since $R$ is prime, every element of $N^{*}(R)$ is a unit of $Q_{0}(R)$; thus $N^{*}(R)$ is precisely the set of all elements of $Q_{0}(R)$ which induce $X$-inner automorphisms of $R$. We will also use $C^{*}(R)$ to denote the nonzero elements in the extended center $C$ of $R$, and $U(R)$ to denote the group of units of $R$.

Theorem 2 suggests the following definition: Let $R$ be prime with derivation $d$. We define $N^{d}(R)=\left\{q \in N^{*}(R) \mid q^{-1} d(q) \in R\right\}, \quad C^{d}(R)=N^{d}(R) \cap C(R)$, and $C^{0}(R)=C^{*}(R) \cap \operatorname{Ker} d$. We may now restate Theorem 2 as follows:

TheOREm $2^{\prime}$. Let $R$ be a domain with derivation $d$, and let $A=R[x ; d]$. Then $N^{d}(R) \subset Q_{0}(A)$ and $N^{*}(A)=C^{*}(A) N^{d}(R)$.

We remark that it is false in general that $Q_{0}(R) \subset Q_{0}(A)$. For, let $A=A_{1}$, the first Weyl algebra; $A=R[x ; d]$ where $R=k[y]$ and $d(y)=1$. Then $Q_{0}(A)=A$ since $A$ is simple, but $Q_{0}(R)=k(y)$. the rational functions in $y$. Thus $Q_{0}(R) \not \subset$ $Q_{0}(A)$.

Now let $X$-inn $R$ denote the group of all $X$-inner automorphisms of $R$, and $\operatorname{Inn} R$ the usual group of inner automorphisms. Writing $N^{*}=N^{*}(R), U=U(R)$, and $C^{*}=C^{*}(R)$ for simplicity, it is clear that for any prime ring $R$ (using $U / C^{*} \cap U \cong$ $\left.U C^{*} / C^{*}\right)$, we have $X$-inn $R \cong N^{*} / C^{*}$, Inn $R \cong U / C^{*} \cap U$, and $X$-inn $R / \operatorname{Inn} R \cong$ $N^{*} / U C^{*}$

We can now obtain our description of $X$-inn $A$.

Corollary 1. Let $A=R[x ; d]$ where $R$ is a domain. Let $N^{*}, C^{*}, N^{d}, C^{d}, C^{0}$, and $U$ denote the subsets of $Q_{0}(R)$ defined above. Then

(1) $X$-inn $A \cong N^{d} / C^{0}, \operatorname{Inn} A \cong U / C^{0} \cap U, X$-inn $A / \operatorname{Inn} A \cong N^{d} / U C^{0}$, 
(2) $X$-inn $A$ is an extension of the central subgroup $C^{d} / C^{0}$ by $H=N^{d} / C^{d}$, which is isomorphic to a subgroup of $X$-inn $R$,

(3) $X$-inn $A / \operatorname{Inn} A$ is an extension of the central subgroup $L \cong C^{d} / C^{0}\left(C^{d} \cap U\right)$ by $K=N^{d} / U C^{d}$, which is isomorphic to a subgroup of $X$-inn $R / \operatorname{Inn} R$.

Proof. (1) To see $X$-inn $A \cong N^{d} / C^{0}$, use $X$-inn $A \cong N^{*}(A) / C^{*}(A)$, Theorem 2', the 2nd isomorphism theorem, and the fact that $C^{*}(A) \cap N^{d}(R)=C^{*}(A) \cap$ $C^{d}(R)=C^{0}(R)$. Since $U(A)=U(R), \quad C(A) \cap U(A)=C^{0}(R) \cap U(R)$, and $\operatorname{Inn}(A) \cong U(A) / C(A) \cap U(A)$, it follows that $\operatorname{Inn} A \cong U / C^{0} \cap U$. The last isomorphism follows from $U / C^{0} \cap U \cong U C^{0} / C^{0}$.

(2) Since $N^{d} \supset C^{d} \supset C^{0}$, it suffices to show that $H=N^{d} / C^{d}$ is isomorphic to a subgroup of $X$-inn $R$. But

$$
N^{d} / C^{d}=N^{d} / C^{*} \cap N^{d} \equiv N^{d} C^{*} / C^{*} \subset N^{*} / C^{*} \cong X \text {-inn } R \text {. }
$$

(3) Using $N^{d} \supset U C^{d} \supset U C^{0}$, it is easy to see $U C^{d} / U C^{0}$ is a central subgroup of $N^{d} / U C^{0}$ since $C^{d}$ is central in $Q(R)$. Define $\phi: U C^{d} \rightarrow C^{d} / C^{0}\left(C^{d} \cap U\right)$ by $\phi(u \alpha)=C^{0}\left(C^{d} \cap U\right) \alpha$, for $u \in U, \alpha \in C^{d}$. Then $\phi$ is well defined, and has kernel $U C^{0}$; thus $U C^{d} / U C^{0} \equiv C^{d} / C^{0}\left(C^{d} \cap U\right)$ by the 2 nd isomorphism theorem. Finally, it is straightforward to check that $N^{d} \cap U C^{*}=U C^{d}$. Thus $N^{d} / U C^{d}=$ $N^{d} / N^{d} \cap U C^{*} \cong N^{d} U C^{*} / U C^{*}=N^{d} C^{*} / U C^{*}$, since $U \subset N^{d}$. This is clearly a subgroup of $N^{*} / U C^{*} \cong X$-inn $R / \operatorname{Inn} R$.

We give an example showing that $A$ can have $X$-inners arising from both $H$ and $C^{d}$. as in (2) of the corollary.

EXAMPLE. Let $k$ be a field and $R=k[G]$, the group algebra. where $G=\langle b\rangle \rtimes\langle a\rangle$ with $a^{-1} b a=b^{-1} . R$ is a domain. with center $Z$ spanned by all $\left\{a^{2 h}\left(b^{\prime}+b^{-1}\right)\right\}$. It is known that $C=C(R)$ is the quotient field of $Z$ [2]. We may define a derivation on $k[G]$ by $d\left(a^{\prime} b^{\prime}\right)=i a^{\prime} b^{\prime}$ (this is a "Type I" derivation, as in [11]), and let $A=$ $R[x ; d]$. As in the example of [9]. $\sigma \in X$-inn $R$ is given by $b^{\sigma}=b, a^{\sigma}=a b$. and $\sigma$ is induced by $\alpha=1+b \in N^{*}(R)$. Since $d(\alpha)=0, \alpha \in N^{d}$. and so $\sigma$ extends to an $X$-inner automorphism of $A$. Now let $\beta=a^{2} \in U \cap C^{*}$. and let $\tau \in X$-inn $A$ denote conjugation by $\beta$. $\tau$ is trivial on $R$, but $x^{\top}=\beta^{-1} x \beta=x+2$. Thus $\tau$ arises from $C^{\prime \prime} / C^{\prime}$.

We note that in this example $C^{(1}(C \cap U)=C^{d}$. so $X$-inn $A / \operatorname{Inn} A$ is isomorphic to a subgroup of $X$-inn $R / \operatorname{Inn} R$. This is not true in general, however, as is shown by Example 1 of (I), where $A=k[y, z][x ; d]$ with $d(y)=y, d(z)=z$. In that case. $X$-inn $R=\operatorname{Inn} R=(1)$, but $X$-inn $A / \operatorname{Inn} A \equiv X$-inn $A \equiv \mathbf{Z}$.

We now apply Theorem $2^{\prime}$ to iterated Ore extensions. The next corollary is analogous to Theorem 3 of [10], where it was show that for any prime group algebra $A=k[G]$ such that the only units are trivial, $X$-inn $A / \operatorname{Inn} A$ is an abelian group.

Corollary 2. Let $A=R_{0}\left[x_{1} \ldots \ldots x_{n} ; d_{1} \ldots d_{n}\right]$ be an iterated Ore extension over the domain $R_{0}$ (that is, $R_{1}=R_{1-1}\left[x_{1} ; d_{1}\right], i=1 \ldots, n$, and $A=R_{n}$ ). Let $s \in N(A)$. Then $s=c_{n} c_{n}, \cdots c_{1} q$, where each $c_{1} \in C\left(R_{i}\right)$, the extended center of $R$, and $q \in N\left(R_{0}\right)$. In particular if $N\left(R_{0}\right)$ is abelian, then $N(A)$ is abelian. 
Proof. The first part of the corollary follows immediately from the theorem, by induction. Now since $c$, commutes with all $c$, for $j \leqslant i$, all the $c$, commute, and commute with any $q \in N\left(R_{0}\right)$. Thus if $N\left(R_{0}\right)$ is abelian, $N(A)$ is abelian.

ADDED IN PROOF. Theorem 1 has also been proved by I. V. Lvov and V. K. Kharchenko, Sibersk. Mat. J. 23 (1982), 193-195. The present author proved this result in February, 1981, and lectured on it at the University of Leeds and at the Hebrew University of Jerusalem in the spring of 1981.

\section{REFERENCES}

1. M. Artin. On Azumara algehras and finite dimensional representations of rings. J. Algchra 11 (1969), 532-563.

2. E. Formanek, Maximal quotient rings of group rings, Pacific J. Math. 53 (1974), 109-116.

3. N. Jacobson, PI-algebras, an introduction, Lecture Notes in Math., vol. 441, Springer-Verlag, Berlin and New York, 1975.

4. V. K. Kharchenko. Differential identities of prime rings. Algcbra i Logika 17 (1978), 220-238 (English transl., 1979).

5. S. Montgomery, Outer automorphisms of semiprime rings, J. London Math. Soc. 18 (1978), 2(19-221.

6. __. Automorphism groups of rings with no nilpotent elements, J. Algebra 60 (1979), 238-248.

7. X-inner automorphisms of filtered algebras, Proc. Amer. Math. Soc. 83 (1981), 263-268.

8. S. Montgomery and D. S. Passman. Crossed products oner prime rings. Isracl J. Math. 31 (1978), 224-256.

10. X-inner automorphisms of group rings. II. Houston J. Math. (to appear).

11. M. K. Smith, Derivations of group algehras of finitely-generated, torsion-free, nilpotent groups. Houston J. Math. 4 (1978), 277-288.

Department of Mathematics. University of Southern California. Los angeles, California 90089 